\title{
Procesos y espacios de investigación en los pregrados de Derecho y Ciencia Política de la sede de Medellín de la Universidad de Antioquia: un diagnóstico entre los años 2004 y 2016
}

\author{
Recibido: 21 de marzo de 2020 • Aprobado: 15 de junio de 2020 \\ https://doi.org/10.22395/ojum.v20n4la7
}

\author{
Lina Claudia Adarve- Calle \\ Universidad de Antioquia, Medellín, Colombia \\ ciderechoypolitica@udea.edu.co \\ https://orcid.org/0000-0002-3750-5913 \\ Ana María Londoño-Agudelo \\ Universidad de Antioquia, Medellín, Colombia \\ ana.Iondonoa@udea.edu.co \\ https://orcid.org/0000-0002-4523-3882 \\ Yesica Alejandra Guzmán-Sossa \\ Universidad de Antioquia, Medellín, Colombia \\ yesica.guzman@udea.edu.co \\ https://orcid.org/0000-0002-1231-4627
}

Investigar es cuestionar y cuestionarse, plantearse interrogantes sustanciales en procesos de ensayo y de error; es la búsqueda del sentido, la articulación de las ideas, el desarrollo de las argumentaciones. También la investigación implica revisar la historia, el pensamiento y los caminos recorridos por otros $y$ otras, relacionarse con las fuentes de información, conocerlas, conquistarlas, perderles el miedo. Investigar es, en sintesis, lograr la sistematización de la experiencia, develar misterios y proyectar sueños. Es colonizar una parcela pequeña o grande, sembrar, cultivar, cosechar y distribuir sus frutos, para que luego vengan otros a mejorar, optimizar y superar nuestra faena.

(Rico, 1996, p. 2)

\section{RESUMEN}

Este artículo tiene por objetivo principal presentar el diagnóstico de los espacios y procesos de investigación en los pregrados de Derecho y Ciencia Política de la Universidad de Antioquia entre los años 2004 y 2016. A partir de un análisis cualitativo de los datos, se identifican los semilleros de investigación y las convocatorias para proyectos de investigación como las principales fortalezas. Además, se hallan como principales retos el fortalecimiento de los grupos de investigación y la consolidación de una comunidad académica. Este diagnóstico es un insumo para el diseño e implementación de políticas, programas o proyectos direccionados a la consolidación, sostenibilidad y fortalecimiento de la investigación en todos los niveles formativos, y representa un precedente para nuevas investigaciones de los pregrados de derecho y ciencia política en el país.

Palabras clave: procesos de investigación; espacios de investigación; Derecho; Ciencia Política. 


\section{Research Processes and Spaces in the Undergraduate Programs of Law and Political Science at the Medellin Campus of the University of Antioquia: a Diagnosis Between 2004 and 2016}

\section{ABSTRACT}

The main objective of this article is to present the diagnosis of research spaces and processes in the undergraduate programs of Law and Political Science of the University of Antioquia between 2004 and 2016. From a qualitative analysis of the data, the research hotbeds and calls for research projects are identified as the main strengths, and the main challenges are the strengthening of research groups and the consolidation of an academic community. This diagnosis is an input for the design and implementation of policies, programs or projects aimed at the consolidation, sustainability and strengthening of research at all educational levels, and represents a precedent for new research in the undergraduate programs of law and political science in the country.

Keywords: research processes; research spaces; Law; Political Science.

\section{Processos e espaços de pesquisa nos programas de graduação em Direito e Ciência Política no campus de Medellín da Universidade de Antioquia: um diagnóstico entre 2004 e 2016.}

\section{RESUMO}

O principal objetivo deste artigo é apresentar o diagnóstico dos espaços e processos de pesquisa nos programas de graduação em Direito e Ciência Política da Universidade de Antioquia entre 2004 e 2016. A partir de uma análise qualitativa dos dados, os leitos de pesquisa e as chamadas para projetos de pesquisa são identificados como os principais pontos fortes. Além disso, os principais desafios são o fortalecimento dos grupos de pesquisa e a consolidação de uma comunidade acadêmica. Este diagnóstico é uma contribuição para a concepção e implementação de políticas, programas ou projetos que visam a consolidação, sustentabilidade e fortalecimento da pesquisa em todos os níveis educacionais, e representa um precedente para novas pesquisas em direito de graduação e ciência política no país.

Palavras-chave: processos de pesquisa; espaços de pesquisa; Direito; Ciência Política. 


\section{INTRODUCCIÓN}

Este artículo es resultado del proyecto de investigación Los procesos de investigación en la Facultad de Derecho y Ciencias Políticas entre 2004-2016: un análisis desde sus principales rasgos; financiado por la Facultad de Derecho y Ciencias Políticas de la Universidad de Antioquia y adscrito a los grupos de investigación Saber, Poder y Derecho, y Gobierno y Asuntos Públicos de la misma Universidad.

El proyecto tuvo como punto de partida la concepción de la investigación, en su acepción más amplia, como un proceso de generación de conocimiento que representa la posibilidad de hacerse preguntas y configura la actividad mediante la cual el conocimiento avanza y se concretiza el desarrollo de la ciencia. Esta inquietud por el conocimiento se inserta como una de las principales preocupaciones en el ámbito educativo debido a su posicionamiento como un quehacer académico destinado especialmente a las instituciones universitarias ${ }^{1}$. Estas instituciones son, primordialmente, las encargadas de formar profesionales capaces de desarrollar conocimientos encaminados a la satisfacción de las necesidades de la sociedad que, a su vez, son expresión de un compromiso ineludible de su progreso y transformación. No hay que perder de vista que la investigación se constituye en un proceso necesario para las universidades en tanto posibilita actualizar y modificar las disciplinas, ya sea en los cambios de paradigma, la reformulación de sus objetos de estudio o de sus métodos, de manera que se correspondan mejor con la realidad social. Así, la investigación no solo está relacionada con el imperativo de comprender el mundo social, sino que también busca entender en qué medida se retroalimenta el proceso educativo.

La importancia de la investigación se ha asumido, entonces, como una premisa incuestionable, tanto por su capacidad de construir y actualizar el conocimiento como por el hecho de que está inserta en unas formas de organización social y económica que lo exigen como condición indispensable para el desarrollo de la ciencia, la educación y la industria. En este sentido, González (2017) afirma:

La investigación es una de las maneras en que se consolidan las capacidades institucionales con el fin de orientarlas hacia la transferencia del conocimiento y su apropiación social, por esto, en la actualidad, las prácticas discursivas institucionales se dirigen a que la formación y la producción investigativa se articulen con la sociedad y el sector productivo. (p. 2)

En virtud de estas razones, Colombia se ha visto comprometida a asumir esta tarea. Desde el surgimiento de Colciencias ${ }^{2}$ en 1968, se presentaron distintos esfuerzos institucionales por fomentar la investigación a través de políticas públicas

\footnotetext{
1 Es preciso aclarar que las universidades no son el lugar exclusivo donde se da la investigación, pues esta puede realizarse en otras instituciones de otra naturaleza, incluso empresarial.

2 A partir de enero del año 2020 se convierte en Ministerio de Ciencia, Tecnología e Innovación a través de la Ley 1951 (2019).
} 
atinentes a todos los niveles formativos. Es así como la Ley 30 (1992), que dicta las disposiciones respecto a la educación superior en Colombia y las normas referidas al proceso de acreditación universitaria, manifiesta el requerimiento de incluir un componente investigativo hasta el punto de convertirse en uno de los ejes misionales de las universidades públicas. Como lo indica el artículo 19 de la ley en mención, se definen las universidades como "instituciones que acrediten su desempeño con criterio de universalidad en las siguientes actividades: la investigación científica o tecnológica; la formación académica en profesiones o disciplinas y la producción, desarrollo y transmisión del conocimiento y de la cultura universal y nacional" (Ley 30, 1992). Esta ley se encuentra en consonancia con la Ley 1286 (2009) que plantea la investigación en tres ámbitos: como fundamento de la docencia; medio de avance de la sociedad; y soporte de la transferencia social del conocimiento. En este marco, las universidades se encuentran con la exigencia de comprometerse con la generación de un conocimiento que contribuya a la comprensión, construcción y transformación de la sociedad de la que forman parte.

La Universidad de Antioquia no es la excepción. En el plan de desarrollo 20062016, Una universidad investigadora, innovadora y humanista al servicio de las regiones y del país, la Universidad de Antioquia se planteó como visión ser la principal universidad de investigación de Colombia y una de las mejores de América Latina. En este sentido, reconoce la investigación, junto a la docencia, como el eje de la vida académica (Universidad de Antioquia, 2006). Asimismo, define la investigación como fuente del saber y base del trabajo docente, y le da un lugar en el currículo a la vez que le asigna una finalidad muy precisa: "la generación y comprobación de conocimientos, orientados al desarrollo de la ciencia, de los saberes y de la técnica, y la producción y adaptación de tecnología, para la búsqueda de soluciones a los problemas de la región y del país" (Universidad de Antioquia, 2006, p. 23).

La Facultad de Derecho y Ciencias Políticas, en tanto dependencia de la Universidad de Antioquia, se ha articulado a estos ejes misionales al desarrollar múltiples procesos relacionados con la búsqueda, generación y transformación del conocimiento con un sentido crítico y compromiso social. Ahora bien, estos procesos no han sido documentados de una manera sistemática ${ }^{3}$, lo que resulta problemático si se admite la necesidad imperante de potenciar y consolidar la investigación por medio de políticas,

\footnotetext{
3 Aunque no se ha hecho un diagnóstico completo y unificado de los programas, es importante resaltar algunos trabajos referidos a la investigación jurídica en general y en la Facultad (Duque, González, Cossio y Martínez, 2018), la pertinencia de la investigación en el programa de Derecho (Gómez, 2005; Franco, 2015), las metodologías en el pregrado (Lopera, 2009; González y Franco, 2012), representaciones sociales de los investigadores del pregrado de Derecho en la Facultad (González y Martínez, 2013). En el caso de Ciencia Política, se sugiere consultar el trabajo de Obando (2014) y los lineamientos de política de investigación para el pregrado de Rojas y Vásquez (2015), entre otros. Asimismo, se recomienda revisar los informes de autoevaluación de los programas, los cuales representaron un precedente e insumo muy importantes para la investigación Los procesos de investigación en la Facultad de Derecho y Ciencias Políticas entre 2004-2016: un análisis desde sus principales rasgos.
} 
proyectos y programas. Estos proyectos no podrían diseñarse de manera asertiva si no se tiene un conocimiento acerca de cómo se lleva a cabo la investigación, qué espacios están más fortalecidos y cuáles presentan mayores necesidades y retos, lo que implica tener un diagnóstico completo del estado de cosas. Además, no puede obviarse la relevancia que supone que las instituciones autoreflexionen sobre sus propios modos y, en un ejercicio autoconsciente, evalúen la calidad de sus procesos y los retos que los secundan.

Como respuesta a esa necesidad surge el proyecto mencionado inicialmente en el que se enmarca este artículo. De modo que el objetivo principal de este texto es presentar el diagnóstico de los espacios y procesos de investigación en los pregrados de Derecho y Ciencia Política de la Universidad de Antioquia entre los años 2004 y 2016. Esta temporalidad obedece a dos razones. Por un lado, en el año 2004 entra en funcionamiento el pregrado de Ciencia Política, un pregrado que, por su misma naturaleza respecto a sus discusiones disciplinares, tiene un fuerte énfasis investigativo. Por otro lado, el año 2016 marca un punto de corte importante si se tiene en cuenta que a partir de ese momento inició el proceso de implementación del eje de profundización ${ }^{4}$ como parte de la reforma curricular en el pregrado de Derecho, el cual tuvo un margen de tres semestres. Por esta razón, fue necesario establecer metodológicamente ese punto de corte en tanto a la fecha no se tienen datos de la manera en que se han desarrollado estos cursos y si se podrían o no considerar como un espacio de investigación en el programa de Derecho. Sin embargo, para efectos de presentar datos actualizados al lector, se harán algunas referencias a años recientes.

Este esfuerzo por sistematizar, organizar, documentar y reconocer cómo se está llevando a cabo la investigación pretende, por un lado, visibilizar los diversos procesos de investigación que se llevan a cabo en los pregrados de la Facultad de Derecho y Ciencias Políticas (sede Medellín). Y, por otro lado, busca contribuir a la tarea de diseñar proyectos, programas y políticas en pro de la consolidación de los procesos de investigación, para lo cual resulta indispensable tener un diagnóstico. En este sentido, no solo se brinda un aporte teórico, sino también experiencial y metodológico que podría ser referente para las instituciones que proyectan procesos similares y que, en todo caso, requieren de la construcción de diagnósticos.

Este proyecto - y en esa medida los resultados que aquí se esbozan — se inscribió en la tradición hermenéutica, en tanto no solo se estudiaron las distintas formas en las que se ha realizado la investigación en la Facultad de Derecho y Ciencias Políticas (sede Medellín) como datos objetivos y comprobables, sino que también se indagó por las percepciones y los significados sobre la investigación. En este caso, la tradición interpretativa parte de reconocer que "los seres humanos son agentes

4 Según la estructura curricular actual, en los cursos de profundización es donde se despliegan con más fuerza, las herramientas investigativas de las que se han dotado al estudiante en el transcurso de su carrera. 
«significativos», el fin de los investigadores debe ser descubrir los significados que motivan sus acciones" (Della Porta y Keating, 2013, p. 37).

En esta línea, el proyecto se inscribió en un enfoque metodológico cualitativo en tanto partió del reconocimiento de su objeto, los espacios y procesos de investigación, como una construcción sociocultural que es expresión de concepciones epistemológicas y metodológicas frente al objeto de conocimiento: el derecho y la política. Se utilizaron como técnicas de investigación cualitativa la revisión documental, las entrevistas semiestructuradas y los grupos focales. Si bien algunos datos derivados de la primera técnica son cuantitativos, estos no sufrieron un tratamiento estadístico en sentido estricto, sino que se interpretaron a la luz de las categorías de la investigación y los datos hallados en campo en tanto ya habían sido sistematizados en otros informes de la Facultad. La revisión documental consistió en un rastreo de toda la literatura referida al debate en torno a los procesos de investigación, del archivo del centro de investigaciones, los planes de decanatura, los proyectos y planes de los programas. Las entrevistas semiestructuradas se realizaron en clave de profundización y validación y se aplicaron a nueve profesores de los dos pregrados; el criterio de selección usado fue el hecho de que conocieran de primera mano los procesos de investigación. Finalmente, los grupos focales se utilizaron con el fin de validar los datos y las conclusiones. Se realizaron cinco grupos focales con coordinadores de los semilleros de investigación, líderes de los grupos de investigación, estudiantes de los pregrados de Derecho y Ciencia Política, integrantes del área de investigación del pregrado de Derecho y el Comité Técnico de Investigaciones. Además, se realizó una retroalimentación virtual con el área de investigación del pregrado de Ciencia Política.

Una vez aclarada la ruta metodológica que fundamenta los hallazgos que aquí se exponen, se presentan a continuación los resultados. En primera instancia, se abordan las principales categorías que guiaron la investigación. Seguidamente, se presenta el diagnóstico de los espacios y procesos de investigación en cada uno de los pregrados. Por último, se presentan algunas conclusiones y se hace hincapié en las fortalezas y los retos en términos de investigación que este diagnóstico revela.

\section{ABORDAJE CONCEPTUAL. PROCESOS Y ESPACIOS DE INVESTIGACIÓN}

\subsection{Procesos de investigación: ¿investigación formativa, formación investigativa o investigación en sentido estricto?}

El debate en torno a las concepciones sobre la investigación y sus distintos procesos, tales como la investigación en sentido estricto, la investigación formativa y la formación investigativa, no ha cesado y son múltiples las visiones. Por un lado, hay quienes la conciben de manera jerárquica donde la investigación en sentido estricto ocupa la cúspide de la pirámide. Otros, comprenden estos procesos de manera paralela. En este sentido, entienden la investigación formativa y la formación investigativa como un simulacro de la investigación en sentido estricto, es decir, consideran que sigue los 
mismos parámetros y pasos, pero con un nivel de dificultad menor debido a que nunca llega a cumplir los cánones de las comunidades académicas. Esta última concepción es introducida por Bernardo Restrepo (2002), la cual es compartida por el Consejo Nacional de Acreditación CNA, quien afirma:

la investigación formativa crea espacios para las prácticas, familiariza con métodos y técnicas, sirve de laboratorio, de ensayo, de experimentación para promover a aquellos docentes y estudiantes que se dedicarán a la investigación en sentido estricto e integrarán los grupos de investigación. (p. 16)

Ahora bien, estas visiones solo expresan una falta de consenso académico en torno a cómo se concibe la investigación. Esta situación no es problemática, pero invita a seguir repensando y a nutrir estos debates que trascienden un hecho meramente terminológico e interviene, finalmente, la forma en que organizamos académica y administrativamente la investigación.

Frente a esa multiplicidad de visiones, fue necesario aceptar e incorporar la diferenciación entre investigación en sentido estricto, formación investigativa e investigación formativa con el fin de nombrar, distinguir y organizar los procesos que se dan al interior de la Facultad de Derecho y Ciencias Políticas. Ahora bien, esta concepción no se tomó de manera arbitraria y forzada, sino que fue adaptada teniendo en cuenta la visión que tiene la institución sobre estos procesos a partir de lo hallado en los documentos institucionales y en las entrevistas. En este sentido, si bien se acepta la diferenciación entre estos tres procesos de investigación, no se conciben de manera jerárquica como sí lo hace Restrepo (2002) y el CNA 5 . Asumimos, además, que no son autoexcluyentes, sino que, de hecho, pueden coexistir. Esto quiere decir que en la investigación en sentido estricto también hay investigación formativa y en la investigación formativa también se presentan procesos de formación investigativa.

Así las cosas, entendemos la investigación en sentido estricto como aquella que se caracteriza por la producción de nuevo conocimiento, el seguimiento estricto de un protocolo y la existencia de una comunidad académica que garantice el criterio de validez. Asimismo, la investigación en sentido estricto supone la existencia de un proyecto de investigación inscrito formalmente en una institución, ya sea de educación superior - IES - o instituciones de otro tipo que tengan regulados los procesos de investigación.

En contraste con la investigación en sentido estricto, se plantea la investigación formativa ${ }^{6}$ que hace referencia a aquellas actividades que pueden o no incorporar la lógica y los métodos de la investigación. Ahora bien, esto no implica necesariamente el

5 Esto no significa que sea una visión menos o más adecuada, lo que aquí se indica es que son distintas y que, para el caso de la Facultad de Derecho y Ciencias Políticas de la Universidad de Antioquia, así se conciben.

6 En este sentido, Berrouet (2006) afirma que "la Investigación formativa da cuenta del proceso de formación continua por ejemplo cuando a un estudiante se capacita en investigación en sentido estricto, pero no necesariamente produce conocimiento científico y realiza este" (citado en Bolívar, 2013, p. 436). 
desarrollo de proyectos de investigación completos ni la producción de conocimiento nuevo y universal. Se trata de aprender, desde la práctica, lo que implica llevar a cabo actividades investigativas aunque ello no implique necesariamente la existencia de un proyecto de investigación formalmente inscrito. Ejemplo de ello son los semilleros de investigación, en el marco de los cuales se realiza revisión documental y elaboración de fichas de contenido, por ejemplo. Al respecto dicen Peláez y Montoya (2013):

La investigación formativa se emplea en el sistema educativo y en particular en las universidades como recurso didáctico (aunque ella misma logra trascender lo didáctico), le permite al estudiante hacer la reconstrucción de los saberes por medio de la formulación de preguntas y elaboración de referentes teóricos para ampliar la apropiación adecuada de contenidos disciplinares. En otras palabras la investigación formativa pretende que el estudiante corra o eleve sus propias fronteras de conocimiento. (p. 21)

Por su parte, la formación investigativa hace referencia al conjunto de actividades relacionadas con la formación y la docencia, tales como los cursos de metodología de investigación, la lectura y discusión de informes de investigación; actividades que necesariamente siguen la lógica del protocolo de investigación. Ejemplo de ello son los cursos de metodología de investigación, donde se forma a los estudiantes en cuanto a los enfoques teóricos y metodológicos y se les enseña a formular proyectos de investigación siguiendo la lógica del protocolo de investigación.

Es necesario insistir que todos estos procesos se retroalimentan. La formación investigativa posibilita el desarrollo de los conocimientos necesarios para aplicar el método científico, los cuales se ponen en práctica por medio de la investigación formativa. Esta posibilita el manejo de todas las herramientas investigativas para llevar a cabo un proyecto de investigación y, en ese sentido, lograr nuevos desarrollos o nuevos conocimientos, esto es, investigación en sentido estricto (Peláez y Montoya, 2013).

\subsection{Espacios de investigación}

Cada uno de los estos procesos antes señalados ocurren en unos espacios concretos que, para efectos de este artículo, se entienden como la conjunción entre personas (estudiantes, profesores, egresados), momentos, situaciones (pregrado, currículo, extracurrículo) y relacionamientos institucionales universitarios (centro de investigaciones, Colciencias, formación universitaria, extensión, regionalización, CODI), que crean las condiciones para que se dé la investigación como proceso. En estos espacios, los procesos no se dan con una relación de exclusividad, sino que puede haber espacios donde coexistan la investigación formativa, la formación investigativa o la investigación en sentido estricto.

Se encontró, entonces, que los pregrados de Derecho y Ciencia Política de la Universidad de Antioquia cuentan con los siguientes espacios de investigación: las convocatorias de investigación, los semilleros, los cursos y los grupos de investigación. 


\section{DIAGNÓSTICO DE LOS PROCESOS Y ESPACIOS DE INVESTIGACIÓN EN LOS PREGRADOS DE DERECHO Y CIENCIA POLIITICA DE LA UNIVERSIDAD DE ANTIOQUIA}

\subsection{Pregrado de Derecho}

En el pregrado de Derecho, que cuenta con una tradición de más de ciento noventa años, se consideró por mucho tiempo la enseñanza del derecho como una actividad que dependía casi exclusivamente de la experticia profesional de los docentes en el ejercicio profesional derivada de la academia o el litigio. Esta concepción se comprende a partir del hecho de que el derecho se concebía como una ciencia o disciplina respaldada por una enorme tradición que se hallaba en los compendios, tratados y manuales.

En este sentido, la formación de los estudiantes se daba a través de la cátedra magistral. Se dejó por fuera la investigación, entendida en términos estrictos como proyectos de investigación, reflexiones de tipo metodológico y seguimiento de un protocolo. Pero esto no implicó un desconocimiento del abordaje riguroso del objeto de estudio por medio de una revisión dogmática y positivista, ni de los aportes en términos de generación de conocimiento que se producían.

La transformación paulatina de este contexto y la introducción de la reflexión investigativa en la Facultad, constituida en ese entonces solo por el pregrado de Derecho, se dio por la confluencia de varios factores y no estuvo exenta de dificultades. Por una parte, fue determinante la política nacional de investigación promovida por Colciencias y adoptada por la Universidad de Antioquia, donde se estipulaba la exigencia de la formación de grupos de investigación en el marco de la cual se creó el Centro de Investigaciones Jurídicas ${ }^{7}$ como forma de institucionalizar la investigación. En este paso a la investigación "industrializada", se requería entonces una articulación con la política nacional de ciencia y tecnología que establecía metas a largo plazo (Rico, 1996). Este paso significó ciertas rupturas con las formas en que en el pregrado concebía la investigación y los modos de generar conocimiento, lo que hizo que el proceso de adaptación, comprensión y crítica de estos procesos fuera lento (Entrevistados Peudeal, comunicación personal, 16 de octubre de 2018; Peudea3, comunicación personal, 17 de mayo de 2019). ${ }^{8}$

Asimismo, la formación doctoral de profesores en derecho y en otras áreas de las ciencias sociales y la llegada de otros docentes cuya formación de base no era el derecho, no solo contribuyó en gran medida a posibilitar la experiencia investigativa en términos estrictos, sino que motivó el replanteamiento del objeto jurídico:

7 Acuerdo Superior 3 y 4 (1982). El Centro de Investigaciones Jurídicas inició su funcionamiento en enero del año 1984.

8 Como forma de proteger la identidad de las y los entrevistados, no se hará referencia explícita a sus nombres sino que se usará el código Peudea más un número para su identificación. 
pongan a cualquiera de los profes que ya somos más mayores, creo que estábamos viviendo esas contradicciones y no es extraño entonces que muchos de esos profesores cuando tuvieron la oportunidad de hacer posgrados no hicieron sus posgrados en derecho, sino que comienzan hacer sus búsquedas en otras áreas. Entonces los hacen en ciencia política, los van hacer en Derechos Humanos, los van hacer en otras áreas y piensen ustedes en algunos de los que han sido referente del pensamiento crítico acá en Colombia, piensen ustedes en personas como Mauricio García, como Rodrigo Uprimny, piensen en muchos de ellos, en los profes de la Nacional, los profes de los Andes, los profes del Externado. Muchos de ellos estaban haciendo sus posgrados eran en áreas distintas, normalmente ciencia política, normalmente Ciencias Sociales o si estaban haciendo estudios en derecho eran con una perspectiva mucho más crítica, mucho más abierta. (Entrevistado Peudea3, comunicación personal, 17 de mayo de 2019)

De esta manera, se comenzó a reevaluar el paradigma de estudio del derecho en la Facultad y se empezó a comprenderlo como un objeto de estudio siempre en construcción, donde la visión positivista dejó de concebirse como el único acercamiento válido. Esto no significa, según lo aclaran los entrevistados, que dejaran de realizarse, por ejemplo, investigaciones dogmáticas. Por el contrario, indica que el espectro de posibilidades se amplió de forma considerable (Entrevistados Peudeal y Peudea7, comunicación personal, 23 de julio de 2019).

Esta idea del derecho como un objeto de estudio en construcción fue impulsada por la reforma curricular del pregrado (Consejo de la Facultad, 2010, Acuerdo 009), pues ella parte del concepto de derecho como "objeto cultural", es decir, un objeto que se transforma y que se encuentra en constante debate y reformulación, lo que lleva a plantear nuevos temas y problemas de investigación (Entrevistados Peudea3, comunicación personal,17 de mayo de 2019; Peudea7, comunicación personal, 23 de julio de 2019). En este sentido, el pregrado se ha propuesto incrementar la capacidad de indagación de los estudiantes como una competencia básica.

En la reforma curricular la investigación aparece como una competencia en tres niveles: en el saber, en el hacer y en el saber hacer. Para desarrollar la investigación como una competencia en el saber, la reforma curricular planteó los cursos de investigación como el lugar en donde los estudiantes adquirirían "conocimientos básicos sobre las metodologías de investigación académica" (Comité de revisión curricular, 2006, p. 12). Y en tanto una competencia en el hacer, la reforma curricular incluye actividades investigativas como:

buscar información; [o|rganizar y sistematizar información; [a|nalizar: [d] iscernir, diferenciar, asemejar, contrastar; [a|rgumentar: para disentir, afirmar, criticar, convencer, persuadir; [a|plicar: cumplir, decidir, resolver, fallar, proponer, negociar, empatizar, defender, acusar; [c|rear: proponer, descubrir, transformar, generar, profundizar, avanzar, trasponer; Ip|roblematizar el derecho: cuestionar, indagar, interrogar, criticar, sospechar; [i]dentificar diferentes formas de resolver problemas. (Comité de revisión curricular, 2006, p. 12) 
Dentro de las cinco habilidades del saber hacer, las investigativas consisten en: "buscar información; [o|rganizar y sistematizar información; [h]erramientas básicas en investigación; Ip|rofundización de temas; [e|laboración de hipótesis; [p|roblematización" (Comité de revisión curricular, 2006, p. 15).

Es evidente el papel central que se asigna a la investigación formativa en la última versión del plan de estudios, la cual es reiterada en el documento preparado por la Subcomisión de Investigación donde se afirma que el plan de estudios "acoge la investigación formativa o formación investigativa, como enfoque pedagógico" (Subcomisión de Investigación, 2009, p. 1). Además, explica su sentido como sigue:

La investigación, el desarrollo de la capacidad de indagación y búsqueda, y la formación de un espíritu creativo en los estudiantes, son elementos centrales del Proyecto Educativo del Programa. El Programa, desde su diseño curricular, asumió como estrategia pedagógica la formación investigativa o enseñanza a través de la investigación, o docencia investigativa, y desde este punto de vista reconoce la importancia de "formar individuos críticos, inquietos, y motivados por la búsqueda de saberes". (Subcomisión de Investigación, 2009)

\subsubsection{Grupos de investigación}

La mayoría de los profesores vinculados y ocasionales del pregrado de Derecho hacen parte de alguno de los diez grupos de investigación ${ }^{9}$ que actualmente existen en la Facultad. Adicionalmente, se puede identificar que al menos siete de esos grupos se corresponden, de manera directa o más estrecha, con el objeto de investigación jurídica, estos son: Derecho y Sociedad; Saber, Poder y Derecho; Estado de Derecho y Justicias; Estudios Internacionales: Derecho, Economía, Política y Relaciones Internacionales; Estudios de Derecho y Política; Grupo de Investigación sobre Conflictos, Violencias y Seguridad Humana; y Sistema Penitenciario. Sin embargo, todos los grupos son interdisciplinarios y se componen tanto de docentes de Ciencia Política como de Derecho.

A continuación, puede observarse en la figura 1 el comportamiento de los grupos en relación con su número y categorización según las convocatorias de medición de grupos, para entonces convocadas por Colciencias. Estos datos corresponden a los años comprendidos entre 2011-2018 y se tienen en cuenta los informes de gestión disponibles.

9 Para obtener mayor información de los grupos y sus líneas de investigación puede remitirse directamente a los GrupLAC que están disponibles en la web. 


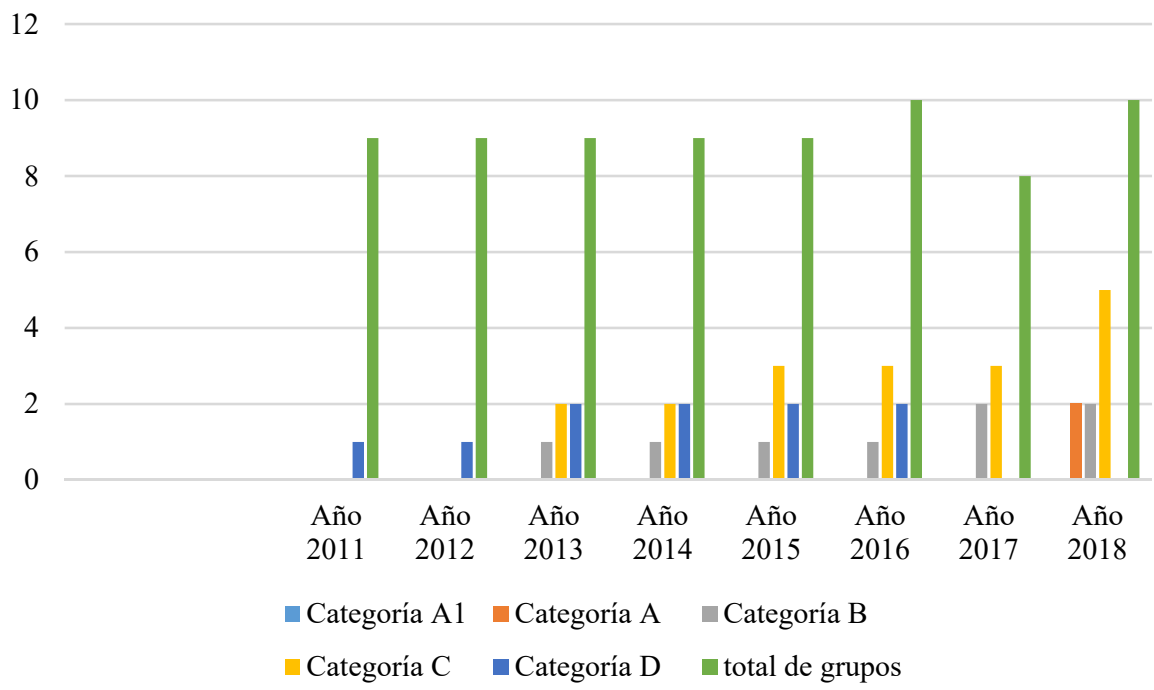

Figura 1. Número de grupos y categorización entre los años 2011-2018

Fuente: elaboración propia a partir de los informes de gestión de la Facultad de Derecho y Ciencias Políticas (2016a) y del Centro de Investigaciones Jurídicas (2018).

Como puede apreciarse en el gráfico, se ha mantenido una cantidad más o menos constante de los grupos de investigación a lo largo de los años que oscila entre ocho y diez grupos, mientras que su categorización en las convocatorias de medición auspiciadas para entonces por Colciencias ha variado significativamente. Para el año 2011 se contaba con un total de nueve grupos, pero solo uno estaba categorizado en D. Para el año 2018 se cuenta con diez grupos, de los cuales dos están en categoría A, dos en B y cinco en C, lo que demuestra una apuesta por la consolidación de los grupos de investigación y un aumento de su productividad reflejado en estas mediciones. Prueba de ello es el aumento de los proyectos de investigación con el aval de estos grupos. Según el informe de gestión del año 2012, los grupos de investigación contaban con dieciséis proyectos de investigación activos (Facultad de Derecho y Ciencias Políticas, 2013), mientras que para el año 2018 se contaban con veintitrés proyectos de investigación activos (Centro de Investigaciones, 2018).

Frente a la dinámica de estos grupos de investigación, los entrevistados expresaron que su percepción es que estos son fundamentalmente una figura administrativa, lo que ven como problemático en tanto no se llega a consolidar una comunidad académica donde prime el conocimiento antes que la burocracia. Esta dinámica se ve reflejada en la concesión de avales que permitan presentarse a convocatorias de investigación, bien sea de la Universidad o de la Facultad, al tiempo que permite a los profesores actuar como investigadores ante el sistema universitario y ante Colciencias (Entrevis- 
tados Peudea3, comunicación personal, 17 de mayo de 2019; Peudea4, comunicación personal, 21 de mayo de 2019; Peudea6, comunicación personal, 14 de mayo de 2019; Peudea7, comunicación personal, 23 de julio de 2019).

En esta línea, los profesores del pregrado, si bien comparten la idea de que es muy importante consolidar una comunidad académica a partir de la comunicación de saberes y la colaboración entre pares, reconocen que en la práctica sigue prevaleciendo la investigación y la formación docente desde el trabajo individual. Además, expresan que la dificultad puede deberse, en parte, a las muchas ocupaciones administrativas y académicas, esto es, a la diversidad de agendas y, más aún, a dificultades propias de las relaciones humanas.

Ahora bien, estas dificultades no representan una imposibilidad fáctica de mediación para la institución. Al contrario, se requiere pensar en estrategias para promover una "cultura organizacional" (Entrevistado Peudea2, comunicación personal, 25 de abril de 2019) y una cultura del encuentro, en donde la discusión, la contraposición de ideas y la validación del conocimiento se conviertan en habitus por medio de la construcción de agendas de investigación comunes que fortalezcan los grupos de investigación.

\subsubsection{Convocatorias}

La Facultad tiene una oferta de convocatorias a nivel nacional con el actual Ministerio de la Ciencia, Tecnología e Innovación (Minciencias) y las demás convocatorias que promueven los convenios interinstitucionales. Además, a nivel universitario, tiene convocatorias programadas por el Comité para el Desarrollo de la Investigación (CODI). Este promueve cada año la convocatoria programática para el área de Ciencias Sociales, Humanidades y Artes, con lo cual se busca una articulación entre la formación de estudiantes de pregrado y posgrado (Universidad de Antioquia, 2017).

Sumado a estas convocatorias, se encuentra el programa Jóvenes Investigadores $\mathrm{U}$ de A. Este programa tiene por objeto que los estudiantes tengan un "entrenamiento en investigación" que les permita conocer experiencias concretas en investigación en el marco de un proyecto de investigación bajo la tutoría del investigador principal y, de esta manera, participar activamente en la dinámica de grupo al que pertenece la investigación (Universidad de Antioquia, 2006). Este es un espacio que ha posibilitado la investigación formativa en los estudiantes de pregrado, y algunos de ellos han derivado sus trabajos de grado de la participación en este programa.

La participación de los profesores del pregrado de Derecho en las convocatorias de investigación universitaria entre los años 2004 y 2016 ha sido promovida institucionalmente. La que registra mayor participación de los profesores ha sido la convocatoria permanente, en la cual la Facultad destina recursos propios. Esto puede explicarse por la flexibilización de los términos de referencia determinados en el marco de las competencias discrecionales y autónomas de la Facultad. 
Sobre la participación de los estudiantes en las convocatorias, se debe señalar que, si bien ha aumentado su participación, este no es muy significativo numéricamente. Esta situación puede relacionarse con varias causas, dentro de las que se destacan: el perfil del egresado que, al ser más amplio, promueve que los estudiantes desarrollen competencias diferentes a las investigativas; el hecho de que no existe un curso de trabajo de grado, lo que hace que las investigaciones relacionadas con trabajo de grado se realicen una vez se tiene el estatus de "egresado no graduado". Además, si bien el plan de estudios del programa de Derecho tiene cursos de formación investigativa y hay cursos en los cuales se aplica la investigación formativa, este último proceso no está muy consolidado en la mayoría de los cursos. Finalmente, si bien la participación del pregrado (profesores y estudiantes) en los semilleros es amplia, la dinámica de los semilleros no está encaminada a la investigación en sentido estricto.

\subsubsection{Semilleros de investigación}

Los semilleros de investigación se han convertido en un espacio predilecto para la investigación formativa, en tanto presentan la posibilidad de repensar las prácticas de formación por medio de diversas metodologías de trabajo y temáticas abordadas. En estos semilleros no se sigue necesaria o exclusivamente la lógica estricta del proyecto de investigación, como sí ocurre en los cursos de investigación.

Asimismo, son un espacio por medio del cual se ha resignificado la relación entre profesores y estudiantes frente al conocimiento, en tanto usualmente se construyen sobre relaciones más horizontales y se constituyen un espacio alterno a las dinámicas propias de las aulas. Allí los estudiantes tienen mayor margen de obra, de propuestas por fuera de la institución universitaria a través de la participación en ponencias en eventos académicos nacionales e internacionales, salidas de campo, entre otros (Entrevistado Peudea3, comunicación personal, 17 de mayo de 2019).

Una de las características de la experiencia en los semilleros en la Facultad no es solo su número, sino la variedad de prácticas, temáticas, estrategias y repertorios de trabajo:

Los semilleros, uno encuentra distintos semilleros en la facultad. Uno encuentra semilleros de la facultad que integran profesionales de distintas disciplinas tendientes a la investigación o a la extensión, hacer trabajos de asesoría, por ejemplo, como el Semillero de Minería, que integra estudiantes de derecho y de otras disciplinas y uno le nota que mira los asuntos que trata de una manera que propiciaría la investigación, incluso más abierta y muy integral. Pero, también hay semilleros que prácticamente son grupos de estudio, que sobre todo se dan en Derecho, que yo diría que pueden ser grupos de estudio, no sé, en distintos temas, pues sin entrar a detallar qué semilleros sí y qué semilleros no, uno encuentra que hay semilleros... por ejemplo, el laboral, que quizá tiene un tipo de estudio que lo lleva más a: "Bueno, ejercitémonos más como posibles abogados en este campo especializado del conocimiento". (Entrevistado Peudea7, comunicación personal, 23 de julio de 2019) 
Ahora bien, la cantidad de semilleros que hay en el pregrado no puede leerse inmediatamente como un aumento de la investigación en el pregrado de Derecho, puesto que, como se ha venido señalando, los semilleros se deben concebir, especialmente, como un espacio para la formación que usa la estrategia de la investigación formativa y se mantiene sobre la base de la voluntad de trabajo de sus integrantes, de su carácter autogestionado, lo cual implica el trabajo en grupo y la disciplina propia de una actividad extracurricular.

\subsubsection{Cursos}

Según la malla curricular, el programa del pregrado de Derecho se divide en los ejes de contexto, fundamentación, complementación, integración y profundización. En este lugar, interesan los cursos de investigación que pertenecen a los ejes de fundamentación y complementación, además de los cursos de profundización que se reúnen en el eje del mismo nombre.

El curso de Investigación I se concentra en la problematización de un tema desde el saber jurídico. A partir de ahí se construye la pregunta y el objetivo de investigación, y se proporciona un panorama general respecto a los enfoques teóricos del derecho. El curso de Investigación II brinda las herramientas metodológicas para el abordaje de la temática definida en el curso anterior y se toma como avance todo lo desarrollado allí. El curso de Investigación III se concentra en la sistematización y el análisis de la información. Finalmente, en el curso de Investigación IV se busca que el estudiante tenga un dominio del proceso de organización, síntesis e interpretación de la información recolectada y su textualización en un discurso académico sustentado empírica y conceptualmente. Por ello, se puede afirmar, en términos generales, que estos cursos contribuyen al desarrollo de habilidades para el diseño de proyectos de investigación, monografías o artículos de revista.

Es importante aclarar que los cursos de investigación no están dirigidos a la elaboración del trabajo de grado, sino al desarrollo de competencias investigativas de los estudiantes. Esto se comprende si se tiene en cuenta que las normas nacionales, las cuales determinan los requisitos para ser abogado en Colombia y en desarrollo de ellas las normas universitarias, establecen que se puede acceder a este título profesional a través de alguna modalidad de trabajo de grado o judicatura. Lo anterior queda a elección del estudiante, por lo cual no podría haber una materia obligatoria que tuviera esta finalidad (Facultad de Derecho y Ciencias Políticas, 2016b).

Por su parte, los cursos de profundización se relacionan de manera más directa con la investigación formativa y la formación investigativa, ya que la investigación no solo es una herramienta pedagógica, sino que se promueve, en la mayoría de estos cursos, la investigación como la forma de producir conocimiento en las materias especializadas del saber jurídico. Si bien es cierto que algunas de estas clases se dictan sobre la base de la cátedra magistral (Entrevistado Peudea7, comunicación personal, 23 de julio 
de 2019), lo cierto es que se ha venido introyectando la idea de que en este nivel de formación y de especialización debe haber un acercamiento al conocimiento a través de la investigación y el trabajo autónomo de los estudiantes. Lo mismo sucede en los cursos del eje de integración. Como se planteaba en los documentos institucionales (Comisión Curricular, 2010), la relación que tienen estos cursos con elementos prácticos de la formación hace necesario el desarrollo de actividades y habilidades investigativas.

En contraste, los cursos del eje de fundamentación y complementación continúan poniendo en práctica la forma de enseñanza tradicional que consiste en la lectura de documentos y la cátedra magistral a cargo del docente. Dado que el mayor número de los cursos del plan de estudios se encuentran en el eje de fundamentación, se puede inferir que, a pesar de la presencia que se reclama de la investigación en los documentos institucionales de discusión del último plan de estudios, la educación y formación en derecho es todavía muy profesionalizante (Entrevistado Peudea7, comunicación personal, 23 de julio de 2019). Ahora bien, según algunos entrevistados esto no tiene que verse necesariamente como problemático en tanto la investigación en el pregrado de Derecho no tiene que constituir un fin en sí mismo, sino que puede configurarse como un medio donde el ejercicio profesionalizante, por ejemplo, litigar, constituya el fin último. En este sentido, se reconoce la importancia de brindar herramientas y posibilidades para el ejercicio de la investigación en el programa, pero ello no tiene que implicar que todos los estudiantes opten por seguir esa orientación.

Frente a los cursos del eje de contexto hay un panorama similar, puesto que son cursos cuyo objeto es encuadrar algunas discusiones alrededor del Derecho en su perspectiva extrasistemática. Se desarrollan en estos cursos, entonces, metodologías donde también tiene mucho protagonismo el profesor para introducir en la formación de los estudiantes conceptos claves que les permitirán estudiar más adelante el derecho como objeto cultural.

\subsection{Pregrado de Ciencia Políitica}

El pregrado de Ciencia Política ha sido un programa con una definida orientación investigativa tanto por las particularidades de la disciplina como por las condiciones institucionales en que surge en la Universidad de Antioquia.

La ciencia política es una disciplina que desde su surgimiento en el siglo XIX ha tenido una preocupación por definir un objeto y un método propio en la búsqueda de una identidad disciplinar demarcada por unas fronteras que le permitan desarrollar la especialización como condición para su desarrollo como disciplina autónoma. Esta situación no le ha llevado al rechazo del trabajo interdisciplinar. Antes bien, ha tenido desde sus comienzos una marcada influencia de otras disciplinas como la sociología, el derecho y la filosofía en su acercamiento a la investigación de los fenómenos políticos. Sumado a esto, el proceso de institucionalización de la ciencia 
política en Colombia "se da gracias a una crisis paradigmática y a una realidad que exige un cambio en la percepción de la investigación y la constitución de la ciencia" (Rubiano, 2012, citado por Obando, 2014, p. 29).

En términos institucionales, este pregrado se inscribe en una universidad pública donde la investigación, junto con la docencia y la extensión, constituyen los ejes centrales de su misión. Además, el pregrado nace con el apoyo del Instituto de Estudios Políticos cuyo fin primordial lo constituye la investigación, lo que no solo ha influido de manera notable en la importancia que se le da a esta, sino también en las agendas temáticas del pregrado referidas al contexto sociopolítico del país.

Todas esas condiciones disciplinares e institucionales han configurado la ciencias políticas como un pregrado que otorga una centralidad a la investigación. A continuación, se presentan los espacios donde se dan cada uno de los procesos de investigación que ya se han enunciado. Para ello, se tomó como referencia especialmente el documento Lineamientos para una política de investigación elaborado en el año 2017 el cual representa un avance importante respecto al diagnóstico del pregrado en materia de investigación.

\subsubsection{Grupos de investigación}

Entre los espacios donde se lleva a cabo la llamada investigación en sentido estricto se encuentran los grupos de investigación. La Facultad contaba para el año 2018 con un total de diez grupos de investigación. Sin embargo, son específicamente tres grupos ${ }^{10}$, a saber, Gobiernos y Asuntos Públicos; Gobierno, Instituciones y Transparencia; Poder y Nuevas Subjetividades. Otros lugares de lo político que cuentan con una mayor afinidad

10 Para acceder a la información completa de los grupos de investigación puede accederse a los GrupLAC que se encuentran disponibles en la web.

Derecho y Sociedad: https://scienti.minciencias.gov.co/gruplac/jsp/visualiza/visualizagr. jsp?nro =00000000001789

Gobierno y Asuntos Públicos: https://scienti.minciencias.gov.co/gruplac/jsp/visualiza/visualizagr. jsp?nro $=00000000006667$

Saber, Poder y Derecho: https://scienti.minciencias.gov.co/gruplac/jsp/visualiza/visualizagr. jsp?nro =00000000008985

Estudios de Derecho y Política: https://scienti.minciencias.gov.co/gruplac/jsp/visualiza/visualizagr. jsp?nro $=00000000014854$

Grupo de Investigación sobre Conflictos, Violencias y Seguridad Humana: https://scienti.minciencias.gov. co/gruplac/jsp/visualiza/visualizagr.jsp?nro=00000000001812

Gobierno, Instituciones y Transparencia: https://scienti.minciencias.gov.co/gruplac/jsp/visualiza/visualizagr. jsp?nro $=00000000014425$

Poder y Nuevas Subjetividades. Otros lugares de lo político: https://scienti.minciencias.gov.co/gruplac/ jsp/visualiza/visualizagr.jsp?nro=00000000015465

Estado de Derecho y Justicias: https://scienti.minciencias.gov.co/gruplac/jsp/visualiza/visualizagr. jsp?nro $=00000000013039$

Estudios Internacionales: Derecho, Economía, Política y Relaciones Internacionales: https://scienti. minciencias.gov.co/gruplac/jsp/visualiza/visualizagr.jsp?nro $=00000000011377$ 
temática con el pregrado en cuanto sus líneas de investigación refieren a los intereses disciplinares en el abordaje directo de la política como objeto. Ahora bien, como se mencionó en el caso del pregrado de Derecho, los diez grupos son interdisciplinares y profesores de ambos pregrados participan en ellos ${ }^{11}$.

En el marco de estos grupos de investigación, profesores y estudiantes realizan ponencias y asisten a congresos, así como a diferentes encuentros nacionales e internacionales de ciencia política. Caso ejemplar son los tres congresos nacionales de ciencia política organizados y realizados por Accpol en asociación con varias universidades de Bogotá, Barranquilla y Cali, además de otros eventos nacionales como encuentros de estudiantes de Ciencia Política y congresos de disciplinas afines (Facultad de Derecho y Ciencias políticas, 2016a).

\subsubsection{Convocatorias}

En la Facultad se encuentra la convocatoria permanente para financiar proyectos de investigación de profesores vinculados, ocasionales y de cátedra (siempre que cuenten con el aval de un profesor de planta), donde los estudiantes tienen la posibilidad de participar, ya sea en el rol de estudiante en formación o de joven investigador ${ }^{12}$. Gracias a esta convocatoria se han desarrollado cuarenta y siete investigaciones cofinanciadas por la Facultad y el CODI (Facultad de Derecho y Ciencias Políticas, 2016a), lo que evidencia un esfuerzo institucional por disponer de recursos propios para promover y apoyar los procesos de investigación de los profesores.

Por otra parte, se encontró que algunos profesores del pregrado realizan investigaciones en el campo de la ciencia política o en ámbitos cercanos que no están inscritas en el SIIU de la Universidad de Antioquia. Por esta razón, se identifica la necesidad de que estas relaciones colaborativas se hagan de manera institucional y formal para posibilitar la participación y visibilidad de la Facultad en estos procesos, además de que se reconozca la incidencia que todos estos proyectos tienen en la formación de los estudiantes y en los grupos de investigación a partir de los productos que de allí se derivan (Rojas y Vásquez, 2017).

\subsubsection{Semilleros de investigación}

Los semilleros de investigación son el espacio por excelencia en donde tiene lugar la investigación formativa en la Facultad. Ahora bien, según la información arrojada en las entrevistas, se encontró que en la Facultad los semilleros tienen distintos objetivos y desarrollan variadas actividades de acuerdo con estos. En ese sentido, no siempre tienen la investigación formativa como su principal fin, sino que algunos tienen una orientación mayor hacia los procesos de extensión o asesoría.

11 Para recordar la variación del número y categorización de los grupos ver figura 1 de este texto.

12 Según el Informe de Gestión, para el año 2018 se encontraron registrados diecinueve estudiantes con la figura de joven investigador o estudiante en formación en los proyectos de profesores y un total de siete estudiantes con la figura de auxiliares de investigación (Centro de Investigaciones, 2018). 
Uno encuentra semilleros de la facultad que integran profesionales de distintas disciplinas tendientes a la investigación o a la extensión, hacer trabajos de asesoría, por ejemplo, como el Semillero de Minería, que integra estudiantes de derecho y de otras disciplinas y uno le nota que mira los asuntos que trata de una manera que propiciaría la investigación, incluso más abierta y muy integral. Pero, también hay semilleros que prácticamente son grupos de estudio, que sobre todo se dan en Derecho, que yo diría que pueden ser grupos de estudio, no sé, en distintos temas, pues sin entrar a detallar qué semilleros sí y qué semilleros no, uno encuentra que hay semilleros... por ejemplo, el laboral, que quizá tiene un tipo de estudio que lo lleva más a: "Bueno, ejercitémonos más como posibles abogados en este campo especializado del conocimiento". (Entrevistado Peudea7, comunicación personal, 23 de julio de 2019)

Los semilleros son el espacio mejor valorado por los estudiantes en comparación con los grupos de investigación y los grupos de estudio. Según los datos arrojados por la encuesta realizada en el marco del proceso de autoevaluación, un 22,35 \% de los encuestados le otorgaron un puntaje de cinco (Facultad de Derecho y Ciencias Políticas, 2016a).

Así, una de las particularidades de este espacio es la recurrencia y la activa participación por parte de los estudiantes, quienes iniciaron su formación en los semilleros desde el año 2008. Para ese año tenían inscritos dieciséis estudiantes y para el año siguiente, 2009, ya eran cincuenta y cinco. En 2011, la participación de los estudiantes en semilleros seguía estable con un total de cincuenta y ocho. Mientras tanto, en el año 2015, el número de estudiantes de Ciencia Política en semilleros era de cincuenta y uno (Centro de Investigaciones Jurídicas, 2018). Según el Informe de autoevaluación (Facultad de Derecho y Ciencias Políticas, 2016a), los semilleros han apoyado a los estudiantes para encontrar temas de afinidad en la ciencia política, además de promover su espíritu investigativo y crítico al participar de proyectos de investigación y la elaboración de productos como artículos y ponencias que le permiten acceder a estrategias y recursos para la movilidad estudiantil.

La relevancia de este espacio no solo es reconocida por la cantidad de participantes, sino principalmente por sus aportes en términos formativos: profundización de las temáticas y áreas disciplinares, aprendizaje de la investigación desde la práctica, debate de ideas, entre otros. Estos aportes indican, entonces, la necesidad de planear e implementar estrategias que contribuyan a su consolidación y sostenibilidad, de modo que no solo surjan nuevos semilleros, sino que los ya creados se mantengan en el tiempo.

\subsubsection{Cursos}

Los cursos se configuran como un espacio muy importante en el pregrado, especialmente para la formación en investigación. Los cursos del área de formación básica se desarrollan a partir de un problema político a lo largo del semestre, donde el estudiante 
comienza a hacer un ejercicio de delimitación del tema, una ruta para la búsqueda de información. Estos pasos lo van preparando y formando para la investigación (Facultad de Derecho y Ciencias políticas, 2016a).

El área de investigación es transversal a todo el programa y está compuesta por los siguientes espacios de conceptualización: Taller de Escritura, Teoría y Métodos de las Ciencias Sociales, Investigación I y II, Estadística, Diseños Cualitativos, Diseños Cuantitativos, Trabajo de Grado I y II. De este modo, el método de investigación en el pregrado se ciñe a la lógica del proyecto de investigación y se fundamenta en un ciclo de aprendizaje que consiste en complejizar el proceso de manera paulatina en cada uno de los cursos que forman parte de esta área, su discusión con fenómenos de análisis de la Ciencia Política y la continua retroalimentación con los contenidos del currículo.

Según algunos entrevistados, no solo los cursos del área de investigación incentivan el aprendizaje respecto al método, también otros cursos de orientación cualitativa contribuyen a este objetivo, como lo indica uno de los entrevistados:

los ejercicios de clase que demandan que el estudiante no se lea un libro de texto y responda de acuerdo al libro de texto, sino que demandan que el estudiante vaya y lea, vaya consulte prensa, vaya y consulte archivo, vaya y hable con actores; que vaya y a través y de un montón de fuentes y de un método muy básico, que es, bueno, leo, saco unas ideas generales, armo la estructura del ensayo y hago un ensayo. Y uno cree que eso no es investigar, o sea, puede que esa no sea una investigación en términos de: "Bueno, tenía un proyecto y lo ejecuté", no, pero usted sí tiene los elementos básicos de la investigación, usted tenía un tema, usted se trazó un objetivo, y a veces todo eso de manera muy intuitiva, porque la investigación es en sí, yo diría, una actividad humana, o sea, casi que natural al ser humano, el ser humano es curioso, el ser humano se hace preguntas, el ser humano va y descubre, a veces hipotetiza. (Entrevistado Peudea9, comunicación personal, 25 de julio de 2019)

Además, no hay que perder de vista que en estos cursos también se realizan salidas de campo en donde el estudiante tiene la posibilidad de observar, indagar, analizar y plantear nuevos proyectos de investigación que aborden las realidades que allí se encuentran.

\section{CONCLUSIONES}

En este diagnóstico no dejan de ser evidentes las diferencias en torno a las condiciones históricas, institucionales y disciplinares en que surge la investigación en los pregrados de Derecho y de Ciencia Política en la Universidad de Antioquia, a pesar de pertenecer a una misma dependencia académico-administrativa. Sin embargo, es posible rastrear en estas diferencias unos rasgos comunes que dan cuenta de unos espacios y procesos fortalecidos que representan una importancia indiscutible en el 
desarrollo y consolidación de la investigación en la Facultad en general. Entre ellos se pueden identificar los semilleros de investigación y las convocatorias de investigación.

Los primeros representan un espacio para la investigación formativa con una oferta temática muy variada, en donde los estudiantes tienen la posibilidad de profundizar las áreas que son de su interés por medio de las múltiples actividades investigativas que allí se desarrollan. Las convocatorias, por su parte, representan un espacio que es compartido por las demás facultades en tanto son institucionales, pero particularmente la Facultad de Derecho y Ciencias Políticas tiene un potencial en cuanto destina recursos propios para la financiación de una convocatoria permanente donde pueden participar tanto profesores vinculados, como ocasionales y de cátedra. Este espacio resulta fundamental como un vínculo y una promoción directa de la investigación en sentido estricto.

Ahora bien, sin desconocer todos los logros e importancia que tienen los grupos de investigación, es preciso señalar que la mayoría de entrevistados identificaron como un punto crítico la falta de comunicación y cohesión dentro de los grupos de investigación. En este sentido, señalaron la necesidad de encuentros donde se construyan agendas de investigación conjuntas con el fin de que se convierta en un espacio para el debate y la validación del conocimiento, lo que relacionan, a su vez, con la necesidad latente de construir una comunidad académica. Con ello se busca que los grupos no se conviertan en tramitadores de asuntos administrativos, sino que realmente pueda darse una construcción colectiva del conocimiento.

Con relación a ello, también se enfatizó la importancia de definir los roles de quienes coordinan estos espacios, de modo que no solo exista la conciencia de llevar a cabo de la mejor manera posible los procesos de investigación, sino también los procesos administrativos que debe tramitar el grupo al momento de llevar a cabo un proyecto de investigación.

Por lo anterior, se identifican unos retos muy precisos en torno a los grupos de investigación, en términos de construir una verdadera comunidad académica. Se evidencia la necesidad de que estos espacios trasciendan lo administrativo y se constituyan en un espacio para la discusión, el trabajo conjunto y la formulación de proyectos de investigación. Hace falta cultivar una cultura del encuentro y del trabajo articulado con otros grupos.

Por último y en términos más generales, este trabajo ha revelado la importancia de que este tipo de diagnósticos se elaboren a través de metodologías que posibiliten una participación más democrática y, de esta manera, no solo permitan una apertura que recoja las visiones y experiencias institucionales o gubernamentales respecto a la investigación, sino todas las que coexisten dentro de las comunidades académicas. Los resultados arrojaron, precisamente, que al interior de una facultad puede 
haber múltiples visiones sobre la investigación, lo que puede determinar y explicar la constitución de los distintos espacios, así como la forma en que se desarrollan los procesos, incluyendo sus fortalezas y puntos críticos.

\section{REFERENCIAS}

Bolívar, R.M. (2013). Los modos de existencia de la estrategia de semilleros en Colombia como expresiones de la comprensión de la relación entre investigación formativa y la investigación en sentido estricto. Múltiples lecturas, diversas prácticas. El Ágora USB, 13(2), 279-539. https://doi. org/10.21500/16578031.113

Centro de Investigaciones Jurídicas (2018). Informe de Gestión-mayo a diciembre de 2017 [documento inédito]. Facultad de Derecho y Ciencias Políticas, Universidad de Antioquia.

Comisión Curricular, pregrado de Derecho (2010). Propuesta de Plan de Estudios de la Comisión Curricular al Comite de Currículo. Facultad de Derecho y Ciencias Políticas, Universidad de Antioquia.

Comité de Revisión Curricular, pregrado de Derecho (2006, 11 de agosto). Componentes curriculares: objeto, contexto, propósitos de formación, roles y competencias. Facultad de Derecho y Ciencias Políticas, Universidad de Antioquia.

Congreso de la República (1992, 28 de diciembre). Ley 30 de 1992. Por el cual se organiza el servicio público de la Educación Superior. Diario Oficial 40.700. https://www.mineducacion.gov.co/1621/ articles-104539_archivo_pdf.pdf

Congreso de la República (2009, 23 de enero). Ley 1286 de 2009. Por la cual se modifica la Ley 29 de 1990, se transforma a Colciencias en departamento administrativo, se fortalece el Sistema Nacional de Ciencia, Tecnología e Innovación en Colombia y se dictan otras disposiciones. Diario Oficial 47.241. http://www. secretariasenado.gov.co/senado/basedoc/ley_1286_2009.html

Congreso de la República (2019, 24 de enero). Ley 1951 de 2019. Por la cual crea el Ministerio de Ciencia, Tecnología e Innovación, se fortalece el Sistema Nacional de Ciencia, Tecnología e Innovación y se dictan otras disposiciones. https://tic.bogota.gov.co/transparencia/marco-legal/normatividad/ley-1951-2019

Consejo de Facultad (2010). Acuerdo 009 de 2010. Por medio del cual se adopta un nuevo plan de estudios para el programa de derecho de la sede de Medellín. Facultad de Derecho y Ciencias Políticas, Universidad de Antioquia.

Consejo de Facultad (2016). Acuerdo 002 de 2016. Proyecto Educativo del Programa de Derecho (PEP). Facultad de Derecho y Ciencias Políticas, Universidad de Antioquia

Della Porta, D. y Keating, M. (eds). (2013). Enfoques y metodologías de las ciencias sociales: una perspectiva pluralista. Ediciones Akal.Duque, S. P., González, F. P., Cossio, N. A., y Martínez, S. M. (2018). Investigación en el saber jurídico. Editorial Universidad de Antioquia.

Facultad de Derecho y Ciencias Políticas, Universidad de Antioquia (2013). Informe de Gestión 2012. Medellín: Universidad de Antioquia.

Facultad de Derecho y Ciencias Políticas (2016a). Informe de Autoevaluación para la Acreditación de Alta Calidad. Universidad de Antioquia.

Facultad de Derecho y Ciencias Políticas (2016b). Proyecto Educativo del Programa de Derecho. Universidad de Antioquia. 
Franco, E. (2015). Análisis de la pertinencia de la investigación en el programa de derecho de la Universidad de Antioquia. Estudios de Derecho, 72(159), 141-160. https://doi.org/10.17533/udea. esde.v72n159a07

Gómez, G. I. (2005). La investigación, la producción y las prácticas del saber jurídico en la Facultad de Derecho de la Universidad de Antioquia. Estudios de Derecho, 62(140), 93-131. https://revistas. udea.edu.co/index.php/red/article/view/332206

González, F. P. y Franco Gómez, E. (2012). Áreas prioritarias de investigación-APIS-en el saber jurídico, un aporte para la discusión sobre innovación jurídica. Estudios de Derecho, 69(154), 187-205. https:// revistas.udea.edu.co/index.php/red/article/view/18414

González, F. P. (2017). Investigación en el saber jurídico. Análisis del componente investigativo del Área de investigación a partir de las técnicas: ejercicios interpretativos y desarrollo de prácticas reflexivas. Informe final dedicación exclusiva estratégica en docencia. Facultad de Derecho y Ciencias Políticas.

González, P. y Martínez, S. M. (2013). Las representaciones sociales y las prácticas investigativas en el saber jurídico. Opinión Jurídica,12(23), 187-200. https://revistas.udem.edu.co/index.php/opinion/ article/view/575

Lopera, O. L. (2009). Usos de las metodologías de investigación en Derecho. Estudios de Derecho, 66(147), 152-180. https://revistas.udea.edu.co/index.php/red/article/view/2412

Obando, L. M. (2014). La institucionalización de la ciencia política en la Universidad de Antioquia: actores, procesos, logros y desafíos. Facultad de Derecho y Ciencias Políticas.

Peláez, L.E. y Montoya, J. (2013). Investigación Formativa e Investigación en Sentido Estricto: una Reflexión para Diferenciar su Aplicación en Instituciones de Educación Superior. Entre Ciencia e Ingeniería, 7(13), 20-25. https://revistas.ucp.edu.co/index.php/entrecienciaeingenieria/article/ view/644

Restrepo, B. (2002). Conceptos y aplicaciones de la investigación formativa y criterios para evaluar la investigación científica en el sentido estricto. Consejo Nacional de Acreditación, CNA. https://www.epn.edu.ec/wpcontent/uploads/2017/03/Investigaci\%C3\%B3n-Formativa-Colombia.pdf

Rico, A. (1996). Investigación en la universidad colombiana: contexto y estrategias. Nómadas, (5), 1 -9. http://nomadas.ucentral.edu.co/nomadas/pdf/nomadas _ 5/05 _ $12 \mathrm{R}$ _ InvestigacionenlauniversidadColombiana.pdf

Rojas, D.P y Vásquez, A.V. (2017). Lineamientos para una política de investigación. Facultad de Derecho y Ciencias Políticas. Universidad de Antioquia. http://www.udea.edu.co/wps/wcm/connect/ udea/00d238b3-6d70-44 1b-b500-647203618c5d/013_Lineamientos_politica_investigacion. pdf?MOD $=$ AJPERESECVID $=1$ lichEi

Subcomisión de Investigación (2009). Documento para comité de currículo, 26 de mayo de 2009. Facultad de Derecho y Ciencias Políticas, Universidad de Antioquia.

Universidad de Antioquia (2006). Plan de Desarrollo 2006-2016. Una universidad investigadora, innovadora y humanista al servicio de las regiones y del país. http://www.udea.edu.co/wps/wcm/connect/ udea/215c33a8-3909-484c-9b84-c94a8459dc83/plan-dllo-2006-2016.pdf?MOD=AJPERES

Universidad de Antioquia (2017, 19 de abril). Plan de Desarrollo 2017-2026. Una construcción colectiva de la comunidad universitaria. https://bit.ly/33htEYT 